\title{
Endochondral Ossification of Chick Embryonic Femora in vitro and on Chorioallantoic Membrane
}

\author{
Manami Kachi ${ }^{1}$, Toshie Sugiyama ${ }^{2}$ and Seiji Kusuhara \\ ${ }^{1}$ Graduate School of Science and Technology, Niigata University, Niigata 950-2181, Japan \\ ${ }^{2}$ Department of Agrobiology, Faculty of Agriculture, Niigata University, Niigata 950-2181, Japan
}

\begin{abstract}
We observed angiogenesis and endochondral ossification of the femora of chick embryos in vitro, on chorioallantoic membrane (CAM), and in vivo to clarify the cellular processes of avian endochondral ossification. We found that the inside of the in vitro femora was still filled with chondrocytes, despite being cultured for 10 days, while calcification of the diaphysis was not observed. As well, only the cartilage tissue of the epiphysis was enlarged. By contrast, blood vessels invaded into the diaphysis and the marrow cavity was formed in CAM-cultured femora. Cartilage canals extended from the marrow cavity and reached to the resting chondrocyte zone, with normal endochondral ossification occurring as in vivo. This study demonstrates that endochondral ossification occurs in femora in CAM culture similar to that in in vivo femora, but not in in vitro femora. The ossification is dependent on vascular invasion into the embryonic femora. In conclusion, for the endochondral ossification of long bones it is essential to supply the embryo with blood vessels. CAM culture system was found to be a superior endochondral ossification model of the embryonic femur.
\end{abstract}

Key words: chick embryo, chorioallantoic membrane, culture, endochondral ossification, femur

J. Poult. Sci., 45: 51-56, 2008

\section{Introduction}

Bone, an apatite deposition on collagen fibrils, plays an important role as a supportive framework for the body and as a reservoir of calcium and phosphate that are essential for life (Weiner and Price, 1986; Blair et al., 2002). Ontogenesis of bone is mediated by two distinct processes, intramembranous and endochondral ossification.

In intramembranous ossification, osteoblasts are directly differentiated from mesenchymal cells through an abundance of blood vessels in the embryonic period and form flat bones such as the cranium, mandible, and scapula (Palumbo et al., 2003; Xian et al., 2004). By contrast, endochondral ossification is a complicated process that occurs as follows. (1) Mesenchymal cells proliferate and differentiate into prechondroblasts and then into chondroblasts. (2) The chondroblasts secrete the cartilaginous matrix and progressively become chondrocytes embedded within their own matrix, where they lie within lacunae. (3) The chondrocytes progressively become larger, enlarging their lacunae, and the cartilage matrix is calcified. (4) The calcified matrix is resorbed by osteoclasts, and alternately osteoblasts form bone. The sequential processes of this ossification are observed at four distinct zones: the

Received: July 18, 2007, Accepted: August 2, 2007

Correspondence: T. Sugiyama, Department of Agrobiology, Faculty

of Agriculture, Niigata University, Niigata 950-2181, Japan.

(E-mail: sugiyama@agr.niigata-u.ac.jp) resting chondrocyte, the proliferating chondrocyte, the hypertrophic chondrocyte, and the calcified zones; ranging from the epiphysis to the center of the diaphysis (Howlett, 1980; Leach and Gay, 1987; Poole et al., 1989). Endochondral ossification results in the formation of long bones such as the tibia, femur, and humerus. This type of ossification requires blood vessels to supply the progenitors of osteoblasts and osteoclasts (Gerber and Ferrara, 2000). However, angiogenesis in relation to avian endochondral ossification is not fully understood.

The developing chick chorioallantoic membrane (CAM) has been a popular model in vascular biology, especially for the assay of angiogenesis and antiangiogenic responses (Vu et al., 1985; Deckers et al., 2002). The CAM is an immunologically protected area where, with proper techniques, it is possible to observe the development of blood vessels toward implanted tissues in incubated eggs (Eisenstein et al., 1973; Roach, et al., 1998). Thus, it is a suitable culture method for the observation of angiogenesis in various tissues and so it is often used to sequentially study long bone growth. Maeda and Noda (2003) examined the effect of the presence or absence of perichondrium on epiphyseal cartilage growth of 12-day-old embryonic chick tibiae in vitro and in a CAM culture system. In in vitro culture, in contrast to the perichondrium-present tibiae, the perichondrium-absent tibiae represented significant growth of epiphyseal cartilage. This result shows that the perichondrium suppresses chondrocyte proliferation and prevents overgrowth of epiphyseal cartilage. Conversely, 
in the CAM culture system, the growth of epiphyseal cartilage and tibiae total length were similar in the absence or the presence of perichondrium. This indicated that the CAM culture system supplies transplanted tissues conditions close to in vivo ones.

In the present study, to clarify the process of avian endochondral ossification we observed angiogenesis and endochondral ossification of the femur of the chick embryo in vitro, on CAM, and in vivo.

\section{Materials and Methods}

\section{In Vitro Organ Culture of Femora}

Fertilized eggs of White Leghorns were incubated for 9 days at $37^{\circ} \mathrm{C}, 60 \%$ humidity. Femora were excised from 9-day-old chick embryos and adherent muscle carefully removed. Femora were placed on a piece of Lenz paper supported on a stainless steel mesh grid in an organ culture dish, and cultured for up to 10 days in a modification of BGJb medium, which was supplemented with $100 \mathrm{IU} / \mathrm{ml}$ penicillin, $100 \mu \mathrm{g} / \mathrm{m} l$ streptomycin and $100 \mathrm{ml} / \mathrm{L}$ fetal bovine serum. The medium was exchanged every other day, and cultured femora were sampled every other day (Fig. 1A). Femora before culture were termed 0-day cultured femora, and cultured femora termed 2-, 4-, 6-, 8-, or 10day femora, respectively.

\section{CAM Culture of Femora}

Circular windows were opened in the shells of 9-day-old host eggs with a dental drill. The outer shell membrane was removed without damaging the inner shell membrane, CAM or embryonic structures. Femora for transplantation were harvested from 9-day-old chick embryos and grafted immediately on the CAM. The host eggs were sealed and incubated for a further 10 days (Fig. 1B). CAM-cultured femora were sampled in the same way as described for the in vitro experiments. To compare bone growth among in vitro, CAM culture and in vivo, we obtained the femora from 9-, 11-, 13-, 15-, 17-, and 19day-old embryos as controls.

\section{Whole Mount Preparation}

To distinguish between non-calcified cartilage and calcified diaphysis, femora were stained with Alizarin Red S. In brief, whole femora were fixed in $95 \%$ ethanol, and then soaked in $1 \% \mathrm{KOH}$ for 2 days to dissolve soft tissue. After that, they were stained with $0.1 \%$ Alizarin Red S in $1 \% \mathrm{KOH}$, and soaked in $20 \%$ glycerol in $1 \% \mathrm{KOH}$ to remove excess stain. Finally, they were mounted in 100\% glycerol, and the growth of the total length of the femora and the calcified length of the diaphysis were measured with a ruler.

\section{Histological Preparation}

Whole femora were fixed in 10\% phosphate buffer formalin. Some calcified femora at late stages of development and culture such as 15-, 17- and 19-day-old in vivo and 8- and 10-day in CAM culture were decalcified for 24 hours in 10\% EDTA (pH 7.2) after fixation. Thereafter, all femora were dehydrated with a graded ethanol, and embedded in paraffin wax. Sections were cut at 5-7 $\mu \mathrm{m}$
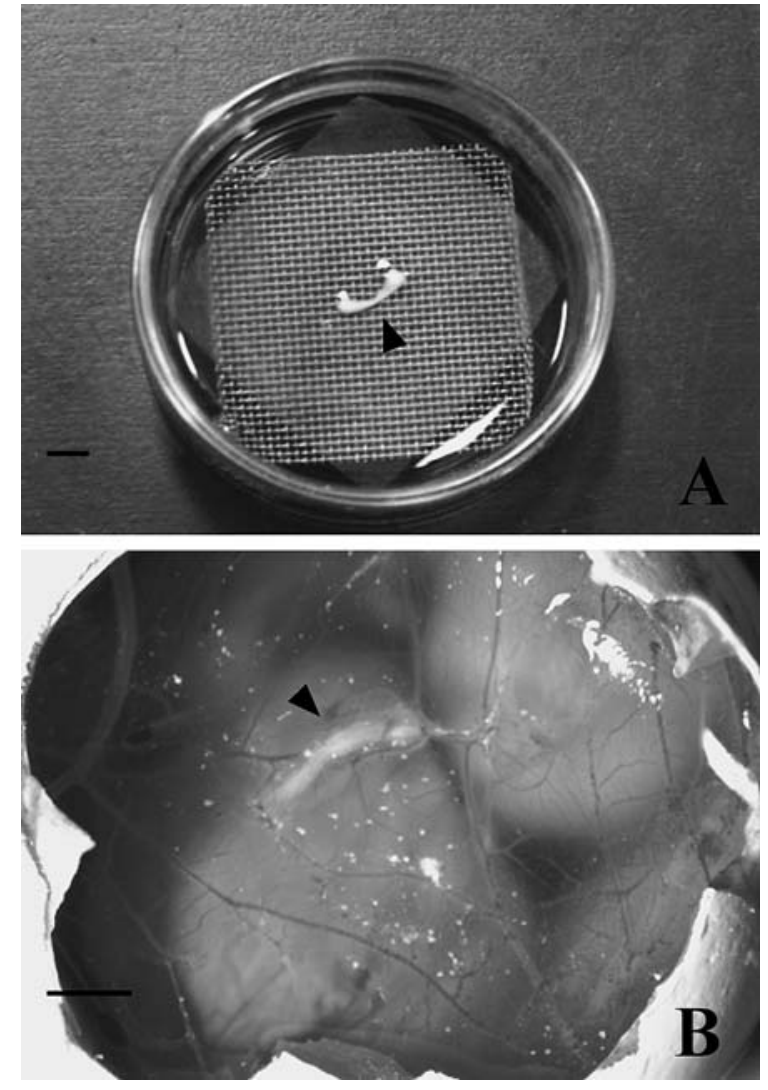

Fig. 1. Culture systems of embryonic femora. Bars $=5$ mm. A: An embryonic femur (arrowhead) cultured for 4 days in vitro. B: An embryonic femur (arrowhead) cultured for 4 days on CAM. The blood vessels of the CAM surround the transplanted femur.

and stained with Hematoxylin-Safranin $\mathrm{O}$ to observe the nuclear and cartilage matrix. Undecalcified sections were also counterstained by von Kossa's method to demonstrate calcium deposits.

\section{Statistical Analysis}

All results were expressed as means \pm SD. The statistical significance of the difference in the data was evaluated by t-test. For each experiment, six femora were used per group.

\section{Results}

\section{Whole Mount Observations}

Growth of the whole femur and the diaphysis in vivo, the calcified diaphysis being stained red by Alizarin Red S, was both prominent and rapid. The femora grew from 4.90 to $23.73 \mathrm{~mm}$, and the diaphysis from 1.61 to 18.50 $\mathrm{mm}$ (Figs. 2A and 3, respectively). By contrast, the growth of the total length of the in vitro femora was poor, from 5.20 to $10.37 \mathrm{~mm}$, during 10 days of culture. The increase in length of the diaphysis was also slight, from 1.70 to $2.39 \mathrm{~mm}$. Only the extension of the epiphyseal cartilage was prominent (Figs. 2B and 3). Although the growth of CAM-cultured femora was slightly delayed com- 

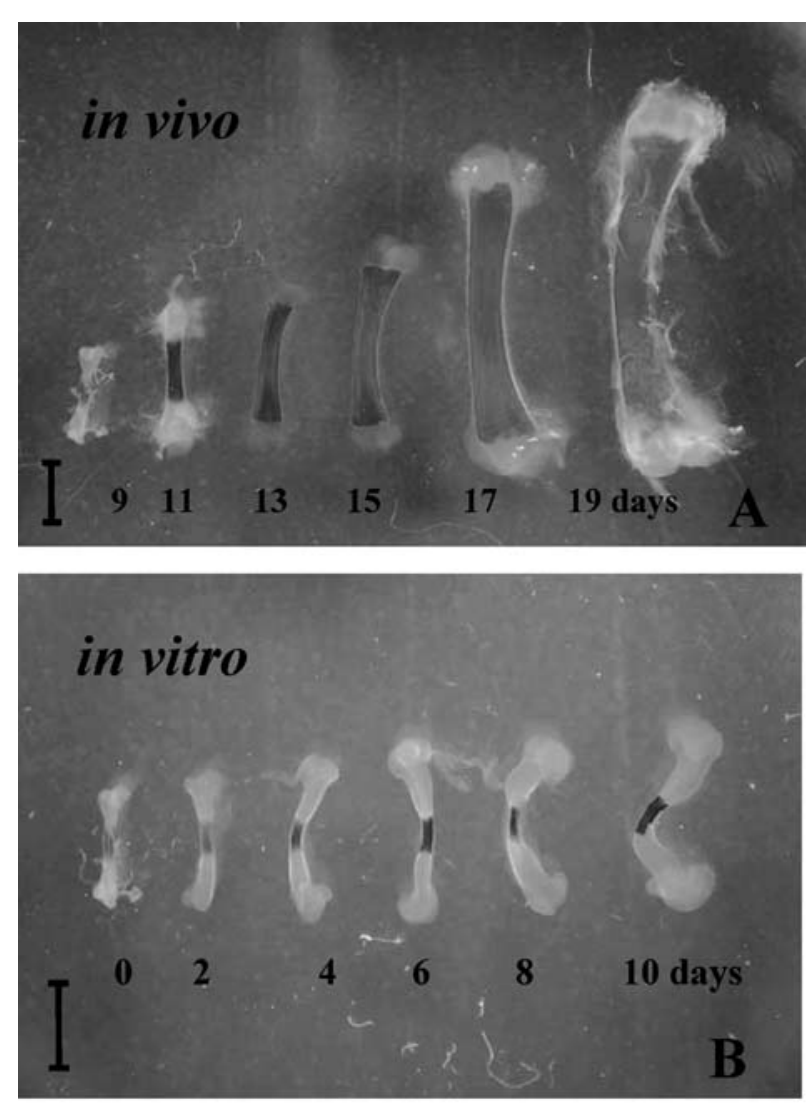

\section{CAM culture}

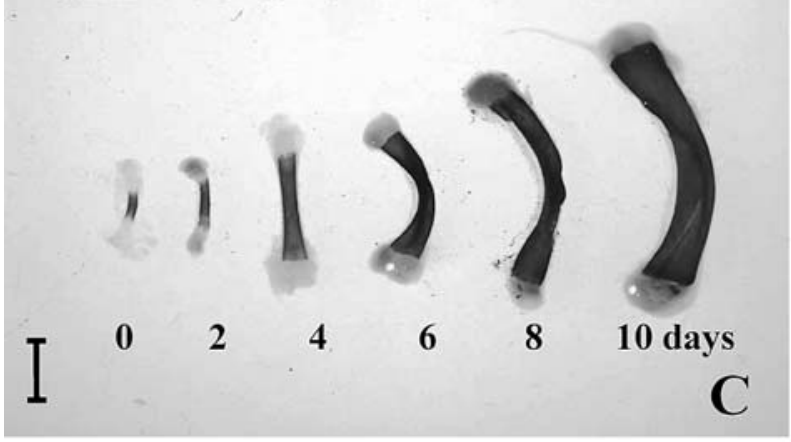

Fig. 2. Whole mount staining of the embryonic femora in vivo (A), in vitro $(B)$ and the CAM culture $(C)$ with Alizarin Red S. Bars $=5 \mathrm{~mm}$. A: Bone growth of in vivo femora. The whole length and the diaphysis show marked growth. B: Bone growth of in vitro femora. The diaphysis growth is less than in vivo, though the epiphyseal cartilage enlarges markedly. C: Bone growth of CAM-cultured femora. The whole length and the calcified diaphysis grow markedly, and this bone growth is close to the in vivo femora.

pared with in vivo, bone growth was prominent, as also observed in the in vivo femora. The lengths of the whole femur and the diaphysis reached $17.87 \mathrm{~mm}$ and $14.07 \mathrm{~mm}$, respectively (Figs. 2C and 3, respectively).

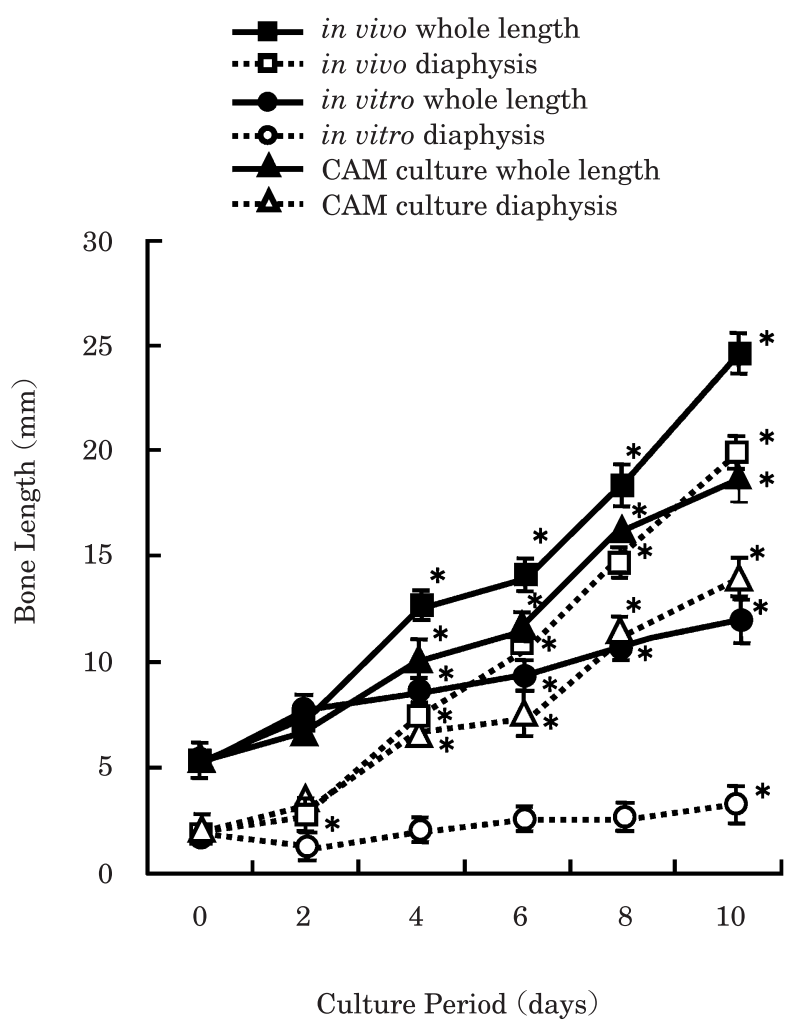

Fig. 3. Growth of embryonic femora of in vivo, in vitro, and CAM-cultured. All results are expressed as means \pm $\mathrm{SD}(\mathrm{n}=6)$. $* P<0.05$ vs 0 days.

\section{Histological Observations}

In in vivo 9-day-old embryonic femora, a bone collar was observed between the periosteum and the cartilage rudiment and osteoclasts were present on the inner surface of the bone collar. Vascular invasion was not observed in either the diaphysis or the epiphysis, which were filled with hypertrophic chondrocytes and cartilage matrix. A distinct chondrocyte zone was seen in the epiphysis.

Thereafter, in 13- to 15-day-old embryonic femora, a calcified trabecular network was observed in the middiaphysis, and a large number of hypertrophic osteoblasts were also seen on the trabecular surface. Many bone marrow cells were observed in the cavities of the trabecular networks. Osteoclasts resorbed the cartilage matrix, adjusting the spongy bone of the diaphysis. In 17day-old embryos, a large marrow cavity was observed in the mid-diaphysis, with the cortical bone surrounding the bone marrow cavities. However, trabeculae were not seen in the mid-diaphysis, and the bone marrow cavity was occupied by marrow cells. In the epiphysis of 19-day-old embryos, the resting, proliferating, maturing, and hypertrophic chondrocyte zones, as well as the calcified zone, were observed. Osteoclasts and osteoblasts were often seen on the surface of the calcified cartilage matrix in the calcified zone, suggesting that bone formation occurred in this area (Fig. 4A). Many cartilage canals accompanying 

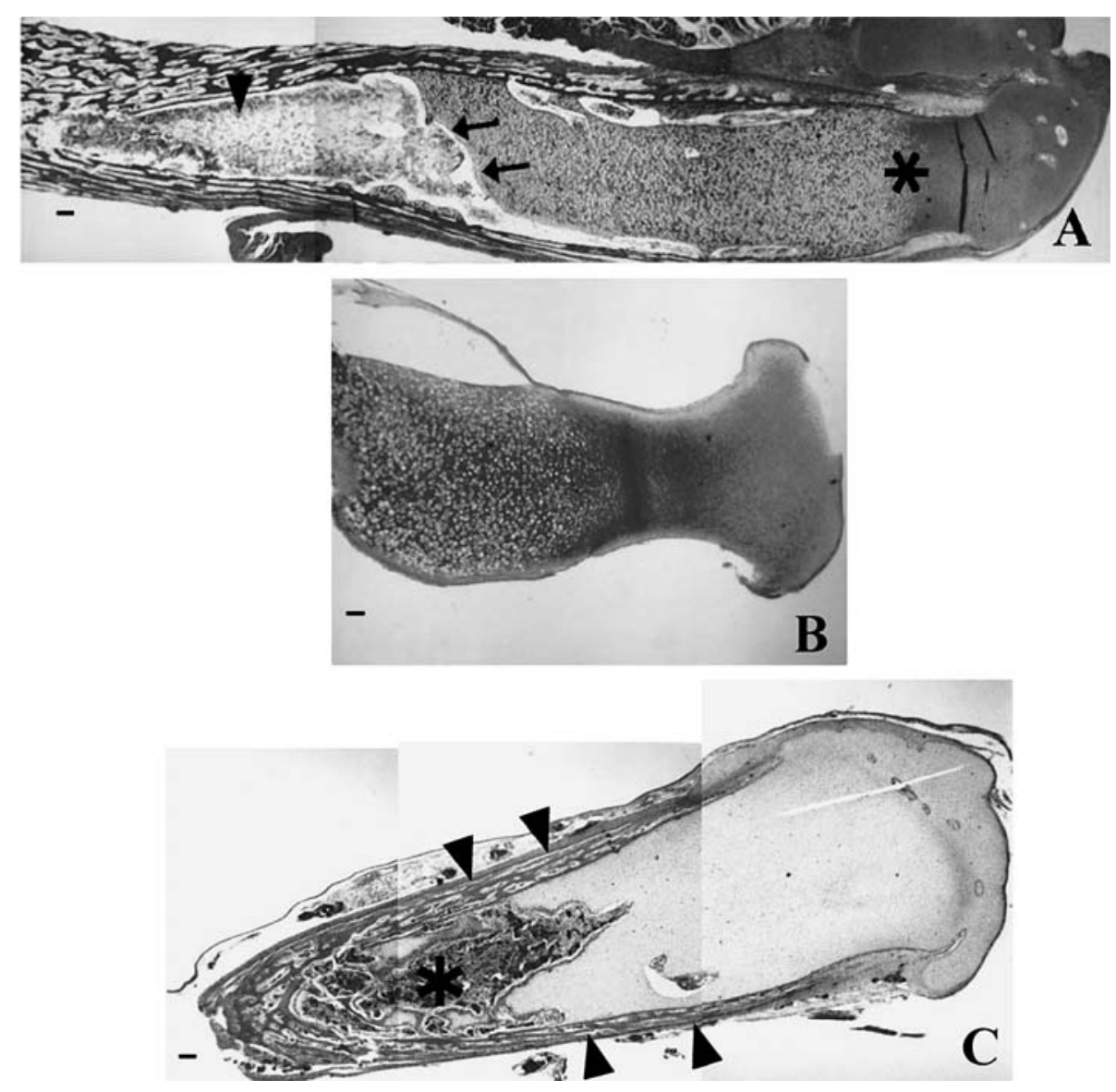

Fig. 4. Histological observations of embryonic femora in vivo (A), in vitro (B) and CAM culture (C). A: In vivo femur of 19-day-old embryo. Formation of a large marrow cavity (arrowhead) is seen, and the cortical bone is observed around it. Moreover, the divisions of the chondrocyte zones (asterisk) are observed. The chondrocytes close to the marrow cavity are hypertrophic, and the cartilage matrix is calcified (arrows). HematoxylinSafranin O. Decalcified section. B: In vitro femur in 10 days. The inside of the bone is filled with chondrocytes, and endochondroral ossification is not observed. Hematoxylin-Safranin O-von Kossa. C: CAM cultured femur in 10 days. The marrow cavity (asterisk) inside the diaphysis enlarge markedly, and cortical bone (arrowheads) is observed around it. Hematoxylin-Safranin O. Decalcified section. Bars $=100 \mu \mathrm{m}$.

blood vessels were seen in the resting zone (Fig. 4A). Around them, chondrocytes represented hypertrophy, and many osteoclasts and osteoblasts were also observed in the cartilage canals. The cartilage matrix in the calcified zone was resorbed by osteoclasts, and the central marrow cavity expanded.

Since the femora before culture ( 0 days in culture) corresponded to those of 9-day-old embryos in vivo, histological observations on the in vitro femora at this stage were equivalent to those mentioned above. After 2- and 4-day in vitro culture periods, the chondrocytes at the center of the diaphysis were more hypertrophic than after 0 days in culture. However, vessel invasion was not seen in the cartilage rudiment. The bone collar of the diaphysis was formed and mineralized, and intramembranous ossification progressed slightly. Microvasculature was also observed in the bone collar. After 6- and 8-day culture periods, hypertrophic chondrocytes were observed not only in the diaphysis but also in the epiphysis, and the whole femora had enlarged slightly. The periosteum had become thick and the matrix was calcified. Osteoclasts were seen at the outside of the bone collar, but their activity was poor. After a 10-day culture period, endochondral ossification had not occurred, and chondrocytes showed prominent hypertrophy in the diaphysis (Fig. 4B). The calcified area between the cartilage matrix and the bone collar was slightly thicker.

After 2 days of CAM culture, the interior of the femora was occupied by chondrocytes and those in the middiaphysis were hypertrophic. Vascular invasion and the formation of cortical bone were not seen. After 4 days in CAM culture, vascular invasion occurred into the center of the diaphysis from the periosteum (Figs. 5A and B). The formation of the marrow cavity started at this area, and calcified cortical bone was formed. After 8 days in CAM culture, many trabeculae were formed as cortical 

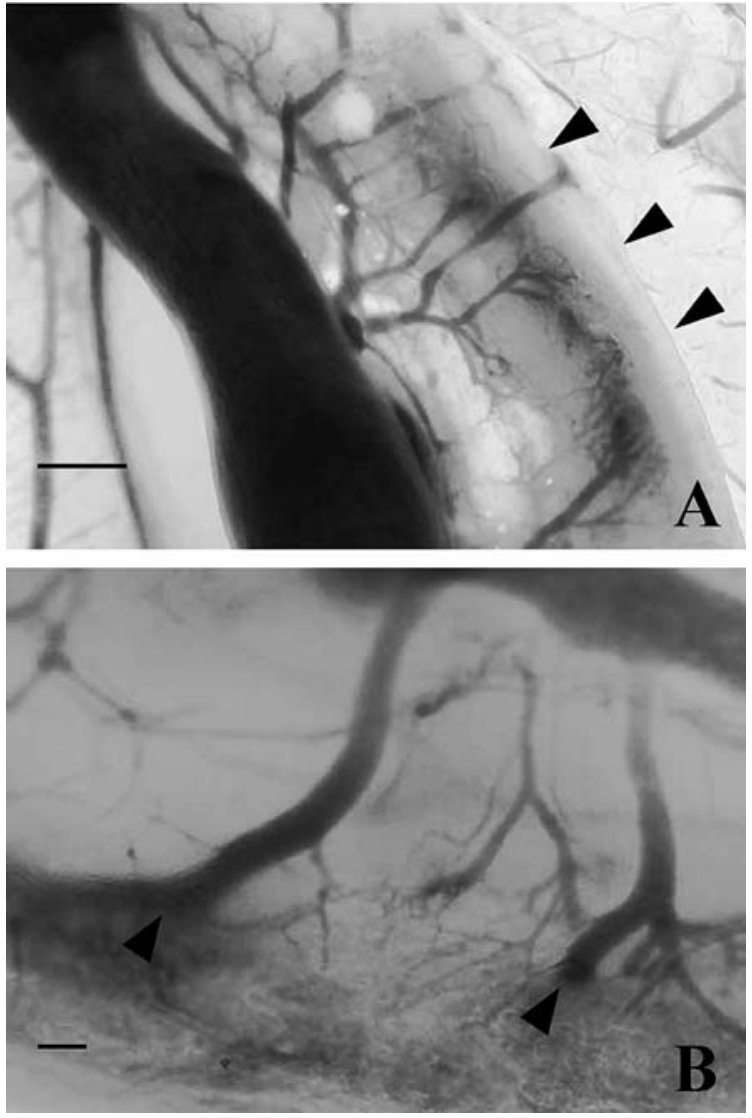

Fig. 5. The invasion of blood vessels upon cultured femur after 4 days on CAM.

A: Femur (arrowheads) cultured for 4 days on CAM. Microvessels derived from the CAM surround the transplanted femur. Bar $=1 \mathrm{~mm}$. B: Blood vessels (arrowheads) from CAM invade the diaphysis of transplanted femur. $\operatorname{Bar}=100 \mu \mathrm{m}$.

bone, and the resting, proliferating, maturing, and hypertrophic chondrocyte zones, as well as the calcified zone, were distinctly observed from the epiphysis toward the diaphysis. Many marrow cells were also present in the marrow cavity. After 10 days in CAM culture, cortical bone of the diaphysis developed and the marrow cavity expanded and was filled with marrow cells. Compared with 8-day CAM culture, the division between the resting and hypertrophic zones was more evident. Many osteoclasts and osteoblasts were observed on the surface of the calcified zone, where bone resorption and formation replaced cartilage matrix with calcified bone matrix (Fig. 4 C). Many cartilage canals accompanying blood vessels invaded from the marrow cavity to the resting zone of the epiphysis. The chondrocytes around blood vessels were hypertrophic. They were also eroded and resorbed by macrophages and osteoclasts in the cartilage canals. Moreover, the osteoclasts in the marrow cavity also resorbed cartilage tissue.

\section{Discussion}

In this study, we cultured chick embryonic femora with in vitro and CAM culture systems and then histologically compared them with in vivo femora. The growth and histological features of the in vitro femora were different from those in vivo. Calcified diaphysis of in vitro femora did not enlarge, while the total length of in vitro femora was slightly increased by the proliferation and hypertrophy of chondrocytes. Femora in CAM culture grew similarly to in vivo femora, though bone growth was slightly delayed compared with that in vivo.

In in vitro femora at 0 days, the bone collar had already calcified, whereas the formation of cortical bone was not observed during culture. Formation of the marrow cavity was not observed and the inside of the diaphysis contained only hypertrophic chondrocytes. These results showed that mesenchymal stem cells are not provided and that the formation of the marrow cavity does not occur in femora in vitro, as previously report (Johnson, 1980). However, thickening of the calcified bone collar and the presence of microvascular structures were observed in this culture. These results suggested that some osteoclasts remaining in the bone collar resorbed the bone matrix; or alternately, osteoblasts formed bone tissue in culture.

In the CAM culture system, which is close to the in vivo environment, though a calcified area in the diaphysis and vascular invasion into bone were observed after 4 days, bone development was slightly delayed compared with that in vivo. This observation suggested that transplantation of 9-day-old embryonic femora onto CAM delayed the invasion of blood vessels compared with that in vivo. Because vascular invasion in chick embryonic bone generally begins at 8-9 days (Rooney and Kumar, 1993), femora dissected from 9-day-old embryos need extra time for reinvasion of the vessels. After 6, 8 and 10 days in CAM culture, formation of the marrow cavity was observed in the diaphysis and bone formation was active in the calcified zone by endochondral ossification, leading to bone growth similar to that seen in vivo. After 10-day of culture on CAM, the cartilage canals accompanying the blood vessels extended from the marrow cavity into the resting chondrocyte zone, and distinct divisions of the chondrocyte zones were observed. Maeda and Noda (2003) studied 12-day-old embryonic chick tibiae subjected to organ culture or on CAM culture. As a result, organ cultured epiphyseal cartilage of tibiae without perichondrium grew more than that of the tibiae with perichondrium. By contrast, CAM culture canceled the inhibitory activity of the perichondrium against bone growth. Thus, CAM supplies essential cells such as osteoblasts, osteoclasts, and marrow cells through the blood vessels and induces endochondral ossification. Although the absence or presence of perichondrium was not investigated here, our study showed similar results regarding the growth of tibia and epiphysis. Namely, Maeda and Noda (2003) showed that the growth of the length of tibia and epiphysis was more prominent in 
CAM culture than in in vitro; however, they did not observe in detail the histological differences of endochondral ossification at the site of the epiphysis among their in vitro, CAM culture and in vivo experiments. Thus they did not pay any attention to blood vessels in the ossification process. Whereas, our histological study demonstrated that endochondral ossification occurs in femora in CAM culture, similar to that in in vivo femora, but not in vitro femora. This process was shown to be dependent on vascular invasion into the embryonic femora, with the CAM culture system supplying blood vessels for the implanted femora. These blood vessels transported mesenchymal stem cells, progenitors of osteoblasts and osteoclasts, into the calcified zone and promoted endochondral ossification. By contrast, the in vitro culture system did not provide for vascular invasion of the femora.

In conclusion, for the endochondral ossification of long bones it is essential to supply the embryo with blood vessels. CAM culture of the embryonic femur is a superior endochondral ossification model.

\section{References}

Blair HC, Zaidi M and Schlesinger PH. Mechanisms balancing skeletal matrix synthesis and degradation. Biochemical Journal, 364: 329-341. 2002.

Deckers MM, Van Beek ER, Van Der Pluijm G, Wetterwald A, Van Der Wee-Pals L, Cecchini MG, Papapoulos SE and Lowik CW. Dissociation of angiogenesis and osteoclastogenesis during endochondral bone formation in neonatal mice. Journal of Bone and Mineral Research, 17: 998-1007. 2002.

Eisenstein R, Sorgente N, Soble LW, Miller A and Kuettner KE. The resistance of certain tissues to invasion: penetrability of explanted tissues by vascularized mesenchyme. American Journal of Pathology, 73: 765-774. 1973.

Gerber HP and Ferrara N. Angiogenesis and bone growth. Trends in Cardiovascular Medicine, 10: 223-228. 2000.

Howlett CR. The fine structure of the proximal growth plate and metaphysis of the avian tibia: endochondral osteogenesis. Journal of Anatomy, 130: 745-768. 1980.

Johnson DR. Formation of marrow cavity and ossification in mouse limb buds grown in vitro. Journal of Embryology Experimental Morphology, 56: 301-307. 1980.

Leach RM Jr and Gay CV. Role of epiphyseal cartilage in endochondral bone formation. Journal of Nutrition, 117: 784790. 1987.

Maeda Y and Noda M. Coordinated development of embryonic long bone on chorioallantoic membrane in ovo prevents perichondrium-derived suppressive signals against cartilage growth. Bone, 32: 27-34. 2003.

Palumbo C, Ferretti M and De Pol A. Apoptosis during intramembranous ossification. Journal of Anatomy, 203: 589598. 2003.

Poole AR, Matsui Y, Hinek A and Lee ER. Cartilage macromolecules and the calcification of cartilage matrix. Anatomical Record, 224: 167-179. 1989.

Roach HI, Baker JE and Clarke NM. Initiation of the bony epiphysis in long bones: chronology of interactions between the vascular system and the chondrocytes. Journal of Bone and Mineral Research, 13: 950-961. 1998.

Rooney $\mathbf{P}$ and Kumar S. Inverse relationship between hyaluronan and collagens in development and angiogenesis. Differentiation, 54: 1-9. 1993.

Vu MT, Smith CF, Burger PC and Klintworth GK. An evaluation of methods to quantitate the chick chorioallantoic membrane assay in angiogenesis. Laboratory Investigation, 53: 499-508. 1985.

Weiner S and Price PA. Disaggregation of bone into crystals. Calcified Tissue International, 39: 365-375. 1986.

Xian CJ, Zhou FH, McCarty RC and Foster BK. Intramembranous ossification mechanism for bone bridge formation at the growth plate cartilage injury site. Journal of Orthopaedic Research, 22: 417-426. 2004. 